\title{
Studies of neutron-induced light-ion production with the MEDLEY facility
}

U. Tippawan ${ }^{1,2}$, S. Pomp ${ }^{2}$, P. Andersson ${ }^{2}$, G. Ban ${ }^{5}$, R. Bevilacqua ${ }^{2}$, V. Blideanu ${ }^{5}$, J. Blomgren ${ }^{2}$, Ph. Eudes ${ }^{6}$, Y. Foucher ${ }^{6}$, A. Guertin ${ }^{6}$, F. Haddad $^{6}$, M. Hayashi ${ }^{2,3}$, C. Lebrun' ${ }^{5}$, F.R. Lecolley ${ }^{5}$, J.F. Lecolley ${ }^{5}$, T. Lefort ${ }^{5}$, L. Nilsson ${ }^{2}$, N. Marie ${ }^{5}$, A. Öhrn², M. Österlund ${ }^{2}$, A. Prokofiev ${ }^{4}$, V. Simutkin ${ }^{2}$, I. Slypen ${ }^{7}$, P.A. Söderström² ${ }^{2}$, and Y. Watanabe ${ }^{3}$

${ }^{1}$ Fast Neutron Research Facility, Chiang Mai University, P.O. Box 217, 50200 Chiang Mai, Thailand

2 Department of Neutron Research, Uppsala University, Box 525, 75120 Uppsala, Sweden

3 Department of Advanced Energy Engineering Science, Kyushu University, Japan

4 The Svedberg Laboratory, Uppsala University, Box 533, 75121 Uppsala, Sweden

5 LPC, ENSICAEN, Université de Caen, CNRS/IN2P3, Caen, France

6 SUBATECH, Université de Nantes, CNRS/IN2P3, France

7 Institut de Physique Nucléaire, Université Catholique de Louvain, Louvain-la-Neuve, Belgium

\begin{abstract}
The growing interest in applications involving high-energy neutrons ( $\mathrm{E}>20 \mathrm{MeV})$ demands high-quality experimental data on neutron-induced reactions. Such data have been measured with the MEDLEY setup at the The Svedberg Laboratory (TSL), Uppsala, Sweden. It has been used to measure differential cross sections for elastic nd scattering and double-differential cross sections for light-ion production $(\mathrm{A} \leq 4)$ with targets ranging from $\mathrm{C}$ to $\mathrm{U}$ and at incident neutron energies around $96 \mathrm{MeV}$. We summarize the experimental results obtained so far and compared with theoretical reaction model calculations. A new method for correcting charged-particle spectra for thick target effects has been used for data obtained with the MEDLEY facility. The new quasi-monoenergetic neutron beam facility of TSL offers the possibility to extend these measurements up to neutron energies of $175 \mathrm{MeV}$. In January 2007, the neutron beam facility at TSL has been equipped with improved shielding and pre-collimator to reduce the background observed with MEDLEY during the first experimental campaigns at $175 \mathrm{MeV}$ to an acceptable level. We present the current status of the MEDLEY facility after the shielding upgrade. We summarize also our ongoing projects including both measurements of light-ion production at $175 \mathrm{MeV}$ from $\mathrm{C}$ to $\mathrm{U}$ targets and fission studies of $\mathrm{U}-238$ in the energy region of 11 to $175 \mathrm{MeV}$.
\end{abstract}

\section{Introduction}

Over the past years development has been made in a wide variety of different applications involving interactions of fast neutrons (20-200 MeV) with nuclei. Examples are dosimetry at commercial aircraft altitudes and in space [1,2] and radiation treatment of cancer within the field of medicine [3], soft-error effects in computer memory within electronics [4], and energy production and transmutation of nuclear waste [5] within energy applications. For all these applications, an improved understanding of neutron interactions is needed for calculations of neutron transport and radiation effects. It should be emphasized that for these applications, it is beyond reasonable efforts to provide complete data sets. Instead, the nuclear data needed for a better understanding must come to a very large extent from nuclear scattering and reaction model calculations, which all depend heavily on nuclear models, which in turn are benchmarked by experimental nuclear reaction cross section data.

The MEDLEY facility [6], located at the The Svedberg Laboratory (TSL) in Uppsala, Sweden, has over the past years performed measurements of double-differential cross sections for the production of light ions by $96 \mathrm{MeV}$ neutrons [6-9]. Recently, we have started a program on measuring angular distributions of fission fragments [10]. The facility has also proven to be a valuable tool in the search for three-body force effects [11]. All these measurements have been performed at the "old" neutron beam at TSL [12]. At this beam, the neutron fluence above $100 \mathrm{MeV}$, where the cyclotron has to operate in
FM mode, becomes too low to collect good statistics within reasonable time and it was therefore decided to construct a new neutron beamline with shorter distance from the neutron production point to the experimental area, thus delivering higher neutron fluxes. This new beamline is in operation since 2004 [13] and opens up the possibility to extend the experimental program and measure neutron-induced reactions at energies up to $175 \mathrm{MeV}$.

\section{Light-ion production studies with MEDLEY}

\subsection{Experiments and typical results}

During the last few years, we have performed a "complete" set of experiments in order to measure double-differential cross sections of the (n,px), (n,dx), (n,tx), (n, $\left.{ }^{3} \mathrm{Hex}\right)$, and $(\mathrm{n}, \alpha \mathrm{x})$ reactions from light to heavy nuclide such as carbon, oxygen, silicon, calcium, iron, silver, holmium, lead and uranium around incident neutron energies of $96 \mathrm{MeV}$. As an illustration, some results for carbon, oxygen, silicon, iron, lead and uranium are presented in figures 1-3. More details can be found in the refs. [6-9]. Note that carbon data are preliminary and calcium, silver and holmium data are under analysis.

Examples of the double differential (n,px) spectra for carbon, oxygen, silicon, iron, lead and uranium are shown at some angles $\left(20^{\circ}, 60^{\circ}\right.$ and $\left.100^{\circ}\right)$ in figures $1-3$. The main difference among the data is found at low energy (below 


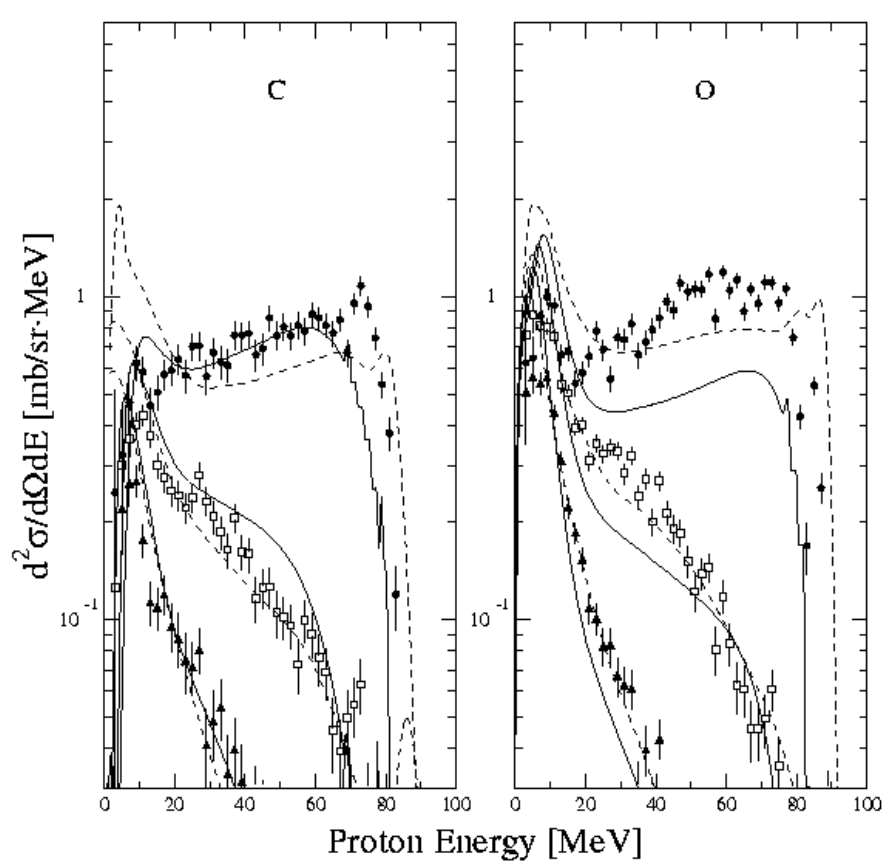

Fig. 1. Experimental double-differential cross sections of the $C(n, p x)$ and $\mathrm{O}(\mathrm{n}, \mathrm{px})$ reactions (left and right, respectively) at $96 \mathrm{MeV}$ at $20^{\circ}$ (filled circles), $60^{\circ}$ (open squares), and $100^{\circ}$ (filled triangles). Solid and dashed curves represent calculations with the TALYS and GNASH codes, respectively.

$20 \mathrm{MeV}$ ) where a compound component is dominant for medium-weight nuclides, i.e., silicon and iron. These lowenergy particles are emitted mainly following the evaporation process of excited nuclei; for carbon and oxygen, the process is less prominent because of low level density while for lead and uranium, this emission is strongly inhibited by the Coulomb barrier. The emission of high-energy protons is strongly forward-peaked and hardly visible in the backward hemisphere. It is a sign of the preequilibrium process. The general trend of the preequilibrium emission becomes dominant with increasing mass number.

\subsection{Comparison with theoretical predictions}

In figures 1-3, the experimental results are presented together with model calculations. The solid lines show calculations with the TALYS code [14] whereas the dashed lines were obtained by the GNASH code [15]. Overall, both predictions give a fair description of the shape of the spectra for all nuclides. At the forward angles $\left(20^{\circ}\right)$, the GNASH predictions give a better description in the mid-energy region for the light to medium-weight nuclide. Note that there is no calculation by GNASH for uranium. The TALYS results account better for the absolute magnitude of the experimental cross sections at large angles for all nuclide while the GNASH calculations overestimate the high-energy parts of the spectra for the medium-weight and heavy nuclides.

For a detailed comparison with theoretical models, angular distributions are needed. In figures 4-5, experimental angular

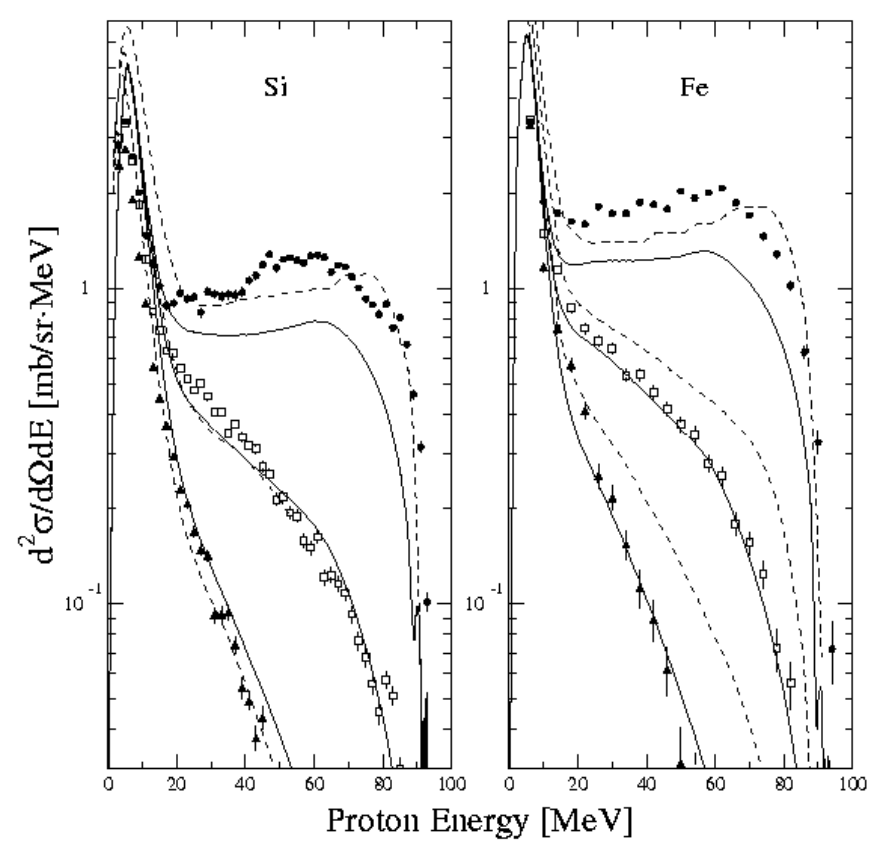

Fig. 2. Same as figure 1, but for $\mathrm{Si}(\mathrm{n}, \mathrm{px})$ and $\mathrm{Fe}(\mathrm{n}, \mathrm{px})$.

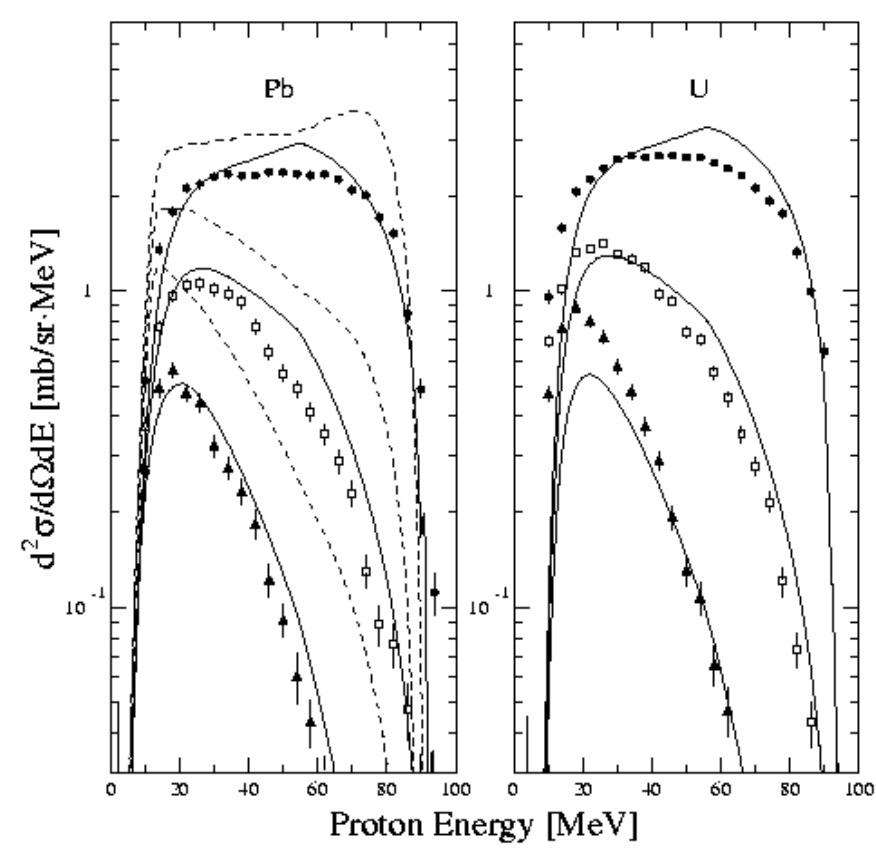

Fig. 3. Same as figure 1 , but for $\mathrm{Pb}(\mathrm{n}, \mathrm{px})$ and $\mathrm{U}(\mathrm{n}, \mathrm{px})$.

distributions at low, medium, and high proton energies for carbon and oxygen, respectively, are shown together with angular distributions calculated on the basis of the TALYS and GNASH models (see ref. [9] for iron, lead and uranium cases). In general, both models give a good description of the data. In the ref. [8], we have compared the experimental data with a preliminary of TALYS code. They show large discrepancies, especially at low energy regions. Using TALY code, version 0.64 , we get a reasonable agreement. 


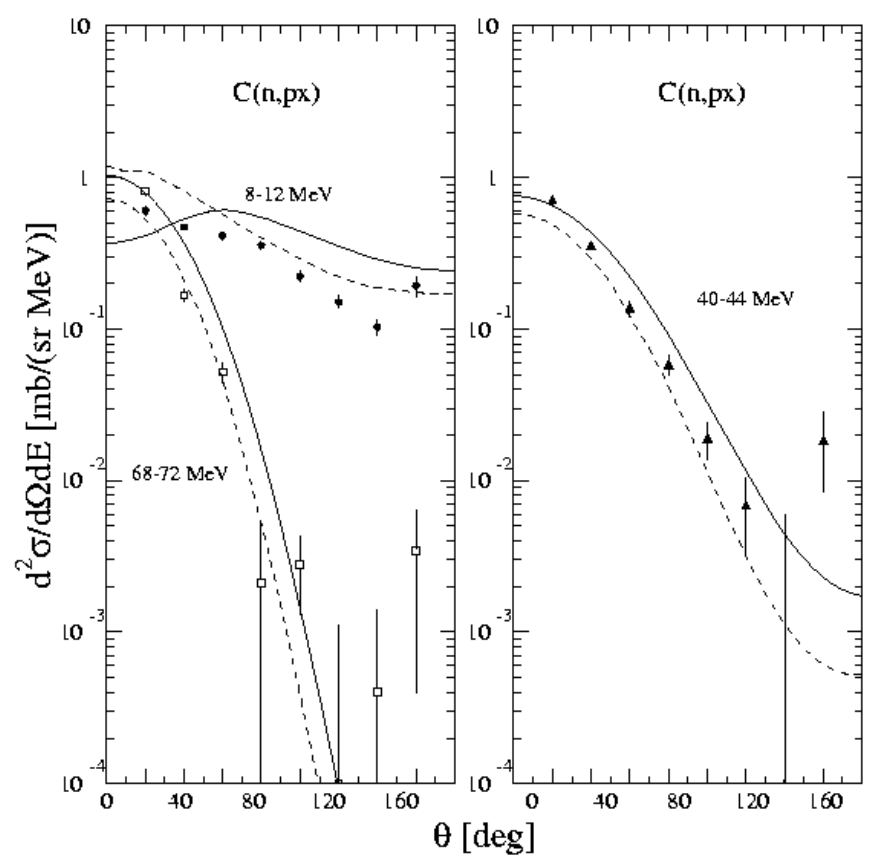

Fig. 4. Angular distributions of $\mathrm{C}(\mathrm{n}, \mathrm{px})$ cross section at ejectile energies of $8-12 \mathrm{MeV}$ (filled circles), 40-44 (filled triangles), and 68-72 (open squares). Solid and dashed curves represent calculations based on the TALYS and GNASH models, respectively.

\section{3 nd elastic scattering}

Neutron-deuteron (nd) elastic scattering in the $60-200 \mathrm{MeV}$ range is one of the most promising ways of investigating threenucleon $(3 N)$ forces. Recent calculations, (see ref. [11] and reference therein), have indicated that the presence of $3 \mathrm{~N}$ forces should appear as a measurable effect in the angular range of the differential cross section minimum.

The nd elastic scattering differential cross section has been performed at $95 \mathrm{MeV}$ incident neutron energy. Models based on inclusion of $3 \mathrm{~N}$ forces describe nd data in the angular region of the cross-section minimum very well, while models without $3 N$ forces cannot account for the data [11] (see fig. 6).

\section{The MEDLEY facility}

The charged particles are detected by the MEDLEY setup [6]. It consists of eight three-element telescopes mounted inside a $90 \mathrm{~cm}$ diameter evacuated reaction chamber. Each telescope has two fully depleted $\Delta E$ silicon surface barrier detectors and one $E$ CsI(Tl) detector. MEDLEY has been equipped with larger CsI detectors to be able to stop protons up to $180 \mathrm{MeV}$. These new detectors have now been used during several runs and perform according to expectations. The CsI crystals have a total length of $100 \mathrm{~mm}$. The first $70 \mathrm{~mm}$ is made cylindrical with a diameter of $50 \mathrm{~mm}$ and the remaining $30 \mathrm{~mm}$ is tapered to $18 \mathrm{~mm}$ diameter to match the size of the readout system. The readout is performed by Hamamatsu S3204-08 photodiodes (PD). The crystals, toghether with the PDs, are mounted inside an aluminum tube and have been
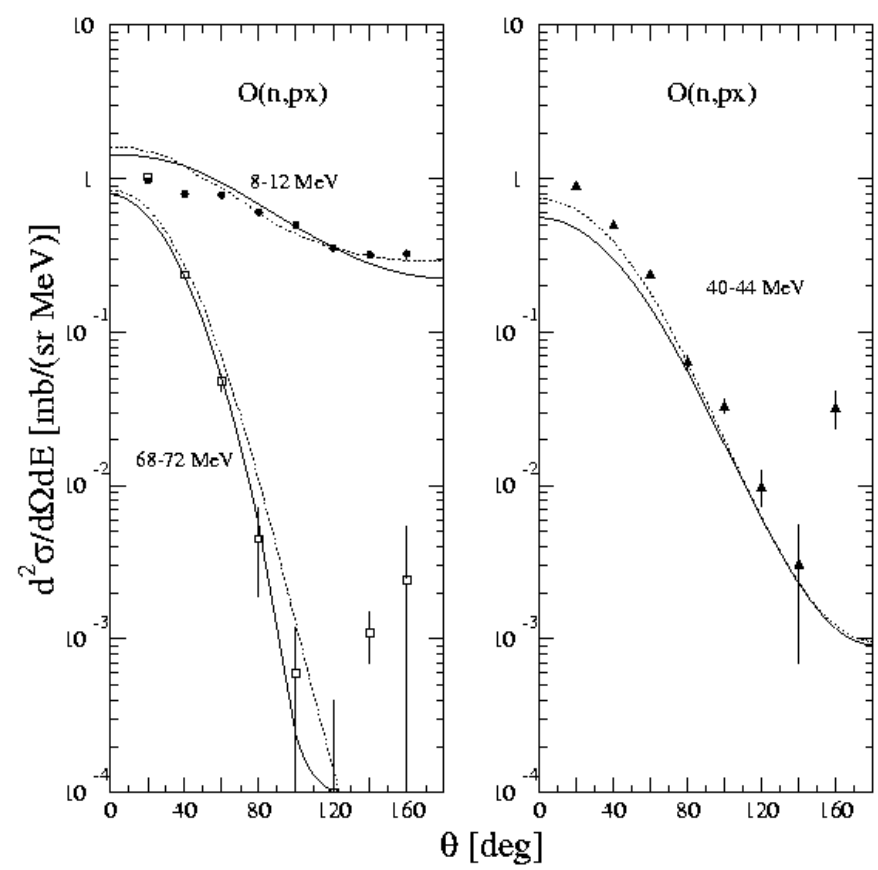

Fig. 5. Same as figure 4 , but for $\mathrm{O}(\mathrm{n}, \mathrm{px})$ cross section.

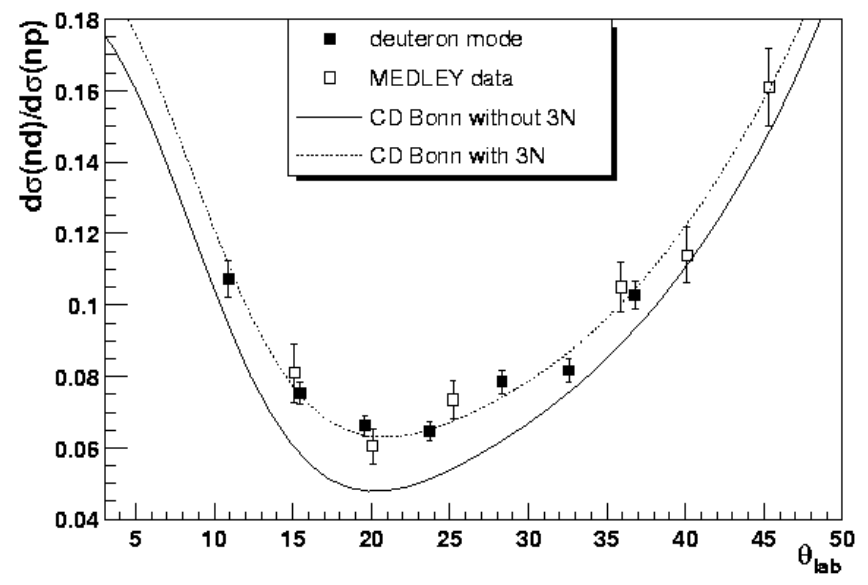

Fig. 6. Ratio of the nd and the np cross sections at $95 \mathrm{MeV}$ as a function of the laboratory angle of the recoiling proton or deuteron [11]. The solid (dotted) line is a cross section calculation, based on the CDBonn nucleon-nucleon potential, without (with) three-nucleon effects included.

manufactured by Saint-Gobain, France. The CsI response function has been tested with $170 \mathrm{MeV}$ proton beam and compared with Monte Carlo simulations, which is decribed elsewhere at this conference [16].

A new method for correcting charged-particle spectra, distorted by energy and particle loss in a thick target [17], has been used for data obtained with the MEDLEY facility [7,8]. It uses an iterative procedure to obtain improved guesses on the inverse response functions for each measured particle energy. The procedure is easy to use, includes a correct treatment of cutoff energies, and has been validated by some test cases. 


\subsection{Background}

During the first runs we have found a rather large background probably due to neutrons from the production target penetrating the concrete shielding. Exchanging the concrete wall to an iron wall has achieved improved background conditions. Available iron blocks from the old CELSIUS ring have actually been used when this reconstruction was undertaken in January 2007. The first data with the new shielding had been taken during February and March 2007. In addition, a pre-collimator, built by holed lead blocks, has been installed temporally inside the clearing magnet. It showed significant improvement of the signal-to-background ratio.

\subsection{Data-taking and analysis}

As mentioned above, we have collected data on ${ }^{12} \mathrm{C}(\mathrm{n}, \mathrm{lcp})$ induced by $175 \mathrm{MeV}$ neutrons early this year. Preliminary double-differential cross sections for carbon are presented in another contribution to this conference by M. Hayashi et al. [16].

\section{Outlook}

Using the MEDLEY facility at the new Uppsala neutron beam, we plan to measure double-differential cross sections for light-ion production on oxygen, silicon, iron, lead, bismuth and uranium at $175 \mathrm{MeV}$. Furthermore we will measure the ${ }^{238} \mathrm{U}(\mathrm{n}, \mathrm{f})$ cross section, together with angular distributions of the fission fragments, over the energy region of 20 to $175 \mathrm{MeV}$. The proposed target nuclei are of highest interest within the applications listed above, and, in addition, of key interest for model development.

\section{References}

1. D. O'Sullivan, D. Zhou, E. Flood, Investigation of cosmic rays and their secondaries at aircraft altitudes, Radiat. Meas. 34, 277 (2001).

2. T.W. Armstrong, B.L. Colborn, Predictions of secondary neutrons and their importance to radiation effects inside the international space station, Radiat Meas. 33, 229 (2001).
3. D.L. Schwartz, J. Einck, J. Bellon, G.E. Laramore, Fast Neutron Radiotherapy For Soft Tissue And Cartilaginous Sarcomas At High Risk For Local Recurrence, Int. J. Radiat. Oncol. Biol. Phys. 50, 449 (2001).

4. Single-Event Upsets in Microelectronics, topical issue, edited by H.H.K. Tang, N. Olsson, Mat. Res. Soc. Bull. 28 (2003).

5. J. Blomgren, Experimental activities at high energies, invited lecture at Workshop on Nuclear Data for Science $\mathcal{E}$ Technology: Accelerator Driven Waste Incineration, Trieste, Italy, Sept. 10 21, 2001.

6. S. Dangtip et al., A facility for measurements of nuclear cross sections for fast neutron cancer therapy, Nucl. Instrum. Meth. Phys. Res. A 452, 484 (2000).

7. U. Tippawan et al., Light-ion production in the interaction of $96 \mathrm{MeV}$ neutrons with silicon, Phys. Rev. C 69, 064609 (2004) and Phys. Rev. C 73, 039902(E) (2006).

8. U. Tippawan et al., Light-ion production in the interaction of $96 \mathrm{MeV}$ neutrons with oxygen, Phys. Rev. C 73, 034611 (2006).

9. V. Blideanu et al., Nucleon-induced reactions at intermediate energies: New data at $96 \mathrm{MeV}$ and theoretical status, Phys. Rev. C 70, 014607 (2004).

10. A. Prokofiev et al., A new facility for high-energy neutroninduced fission studies, Proceedings of the International Conference on Nuclear Data for Science and Industry, AIP Conf. Proc. 769 (Melville, New York, 2005), p. 800.

11. P. Mermod et al., Evidence of three-body force effects in neutrondeuteron scattering at $95 \mathrm{MeV}$, Phys. Rev. C 72, 061002(R) (2005).

12. J. Klug et al., SCANDAL-a facility for elastic neutron scattering studies in the 50-130 MeV range, Nucl. Instrum. Meth. Phys. Res. A 489, 282 (2002).

13. S. Pomp et al., The new Uppsala neutron beam facility, Proceedings of the International Conference on Nuclear Data for Science and Industry, AIP Conf. Proc. 769 (Melville, New York, 2005), 780.

14. ICRU Report 63, International Commission on Radiation Units and Measurements, Bethesda, MD, March 2000.

15. A.J. Koning, S. Hilaire, M.C. Duijvestijn, TALYS-0.64 User Manual, December 5, 2004, NRG Report 21297/04.62741/P FAI/AK/AK.

16. M. Hayashi et al., Measurement of light-ion production at the new Uppsala neutron beam facility, in International Conference on Nuclear data for Science and Technology, 2007.

17. S. Pomp, U. Tippawan, An iterative procedure to obtain inverse response functions for thick-target correction of measured charged-particle spectra, Nucl. Instrum. Meth. Phys. Res. A 572, 893 (2007). 